\title{
Use of the Phoenix system for bacteriology
}

\author{
JENNIFER PERRY, DA MITCHISON, JH DARRELL
}

From the Department of Bacteriology, Royal Postgraduate Medical School, Du Cane Road, London W12 OHS

SUMMARY A dedicated microbiology data processing system with remote batched job entry to an $\stackrel{\vec{\circ}}{\overrightarrow{0}}$ obsolete computer, has been superseded by the inclusion of bacteriology in an on-line interactive $\vec{\omega}$ clinical pathology system which had previously incorporated chemical pathology and haematology. The original Phoenix system has been adapted to allow for the entry of bacteriology data using ? mnemonic codes and to deal with the problems caused by the longer processing time of $\stackrel{\circ}{\circ}$ bacteriology specimens. Particular advantages of the new system include the immediate linkage of $\overrightarrow{\overrightarrow{.}}$ all specimens for each patient and an easy recall and display of results in the laboratories and on the wards.

From 1974 the Bacteriology Department, Royal Postgraduate Medical School, used an already obsolete Elliott 4120 to produce daily reports, cumulative reports and a variety of analysis data. ${ }^{1} \mathrm{By}$ 1978 it was obvious that this computer, which was now 12 years old, although remarkably reliable for its age, was coming to the end of its useful life. No funds were available for another dedicated computer but we were given the opportunity to graft our work onto the Phoenix system. This system was being used successfully by the Chemical Pathology and Haematology Departments on a Computer Technology Modular One Computer with a core memory of $56 \mathrm{~K} 16$ bit words. ${ }^{2}$ Owing to the different requirements of bacteriology it was realised that many modifications would be needed to adapt the system for bacteriology use.

\section{Original Phoenix system}

FILES

The Phoenix system designed and in use for chemical pathology and haematology, consists of a set of three main disc files.

\section{Clinical file}

Each patient has one Patient Identification record (PID) containing the case number, name, age, sex, ward and consultant. After the PID is one record for each of the patient's test requests, kept in order of date and time received. This file is accessible from visual display units (VDUs) throughout the hospital and medical school.

Accepted for publication 28 July 1982
Work file

This file contains the details of specimens currently being processed. Each specimen record has a request $\vec{\varphi}$ number which is a pointer to the same specimenos record on the clinical file. The data are stored in laboratory number order within sets. For the purposeo of chemical pathology, the tests performed in the laboratory are grouped into sets of tests which takeo place on a particular apparatus.

\section{Report file}

A specimen which has had its result entered and is waiting to be reported has a record entered in the? report file. These records are stored in ward order so? that printed reports for one ward are "output" sequentially.

\section{PASSAGE OF REQUESTS THROUGH THESE FILES}

Test requesting

When a specimen is received, a request is entered into the system. This results in a record being created on? the work file for that laboratory number and corresponding record being started on the clinical file under that case number.

Results input

When specimen results are entered, they are put into the work file record.

\section{Listing and authorisation}

When completed, all the results for each set ar listed, and passed to the laboratory staff responsible for the set concerned for checking. When a listing has been checked and the necessary amendments made $\frac{?}{1}$ all the specimens on this list are authorised. This 
authorisation involves the transfer of results onto the clinical file and deletion from the work file. An entry is also made on the report file to indicate that there is a result to print.

\section{REPORTING}

Ward reports are a free format listing of all specimens from each ward which have been authorised since the previous run and are available at $4 \mathrm{pm}$ daily. Cumulative reports are produced for each patient on preprinted stationery. Earlier results are repeated with the addition of the latest results, and this supersedes the previous report. When a section of the sheet is full, instructions are printed to file this sheet permanently in the patient's notes. The next result starts the new page. These cumulative reports are printed every morning.

\section{CULLING}

The clinical file contains data for approximately the previous three months. Earlier work is culled to the history file which is still accessible to the laboratories and wards. A date is entered into the culling program and data are transferred to the history file for one of two reasons:

(a) If a patient has been inactive since the cull date then all his data are transferred to the history file.

(b) If a patient has been active since the cull date then his results are transferred if they have been printed on a completed page of a cumulative report.

\section{Modifications of Phoenix system to include bacteriology}

\section{RESULTS LAYOUT}

Bacteriology data input need a method of encoding the long names of organisms, antibiotics, parts of the body etc. Our staff were already well used to encoding results using short mainly three-character mnemonics which match into an index of all valid codes. ' These are well suited to VDU operation so we wished to continue using the method. The specially written bacteriology results input program accepts data coded on the request sheet as in the following examples:

\section{SW L UPP THI 4P SAU 3P PEN R ERY S CLX S}

$=A$ swab of left upper thigh contains pus $4+$ and has yielded a 3+ growth of Staphylococcus aureus resistant to penicillin but sensitive to erythromycin and cloxacillin.

\section{REF VDR 1 THA D FTA N TPI N}

$=A$ reference laboratory report states that serum for syphilis serology has yielded a VDRL slide test positive neat, a doubtful TPHA and negative FTA and TPI tests.
SITE PHRASES

For pus and mycology specimens it is possible to enter a phrase containing a maximum of four mnemonic words to describe the source of the specimen. Thus a wound swab from the right lower arm is coded as:

\section{WS R LOW ARM}

The input program asks the questions "sample 1," "sample 2," "sample 3," "site" which are answered by mnemonic codes or nil entries.

\section{SPECIAL CODES, SPECIAL NUMBER, ORGANISM AND PROFUSION}

Results for all other specimen types can be coded in the following standard format: "special code," "special number," "organism," "profusion," "sensitivity." Each of these headings contains a different piece of information depending on the specimen type and the VDU asks the question relative to the specimen type as shown in the Table.

\section{Special code}

The answer for "specimen" for a throat swab could be THR (throat) or NOS (nose). For a tuberculosis specimen the answer could be SP (sputum) or U (urine). The answer for "direct smear" could be GNC (Gram-negative coccus).

\section{Special number}

The special number question "Pus" or "AFB" expects a profusion of pus or acid fast bacilli on a scale $0,+$ to ++++ . The question WBC expects a number up to 32000 white blood cells.

\section{Organism}

For most specimens an organism code is entered. For serology specimens this is often a single code such as AFP for Aspergillus fumigatus precipitins but more complex results can be grouped, as for example AGG CAL 16 CPA N which stands for "agglutinins to Candida albicans 1/16 titre and to $C$ parapsilosis negative." To allow for complex serology there is room for up to 10 tests (or organisms) in each specimen result record.

\section{Profusion}

These results include a scale $0,+$ to ++++ for organisms and $\mathbf{N}$ (negative), $\mathrm{D}$ (doubtful), $\mathrm{A}$ (anticomplementary) and titres for serology and levels for antibiotic assay.

\section{SENSITIVITIES}

For specimen types which have sensitivity tests, the results can be entered in one of two ways, either by entering each antibiotic code followed by its sensitivity, such as PEN R ERY S CLX S or by using a 
Questions asked on input of results

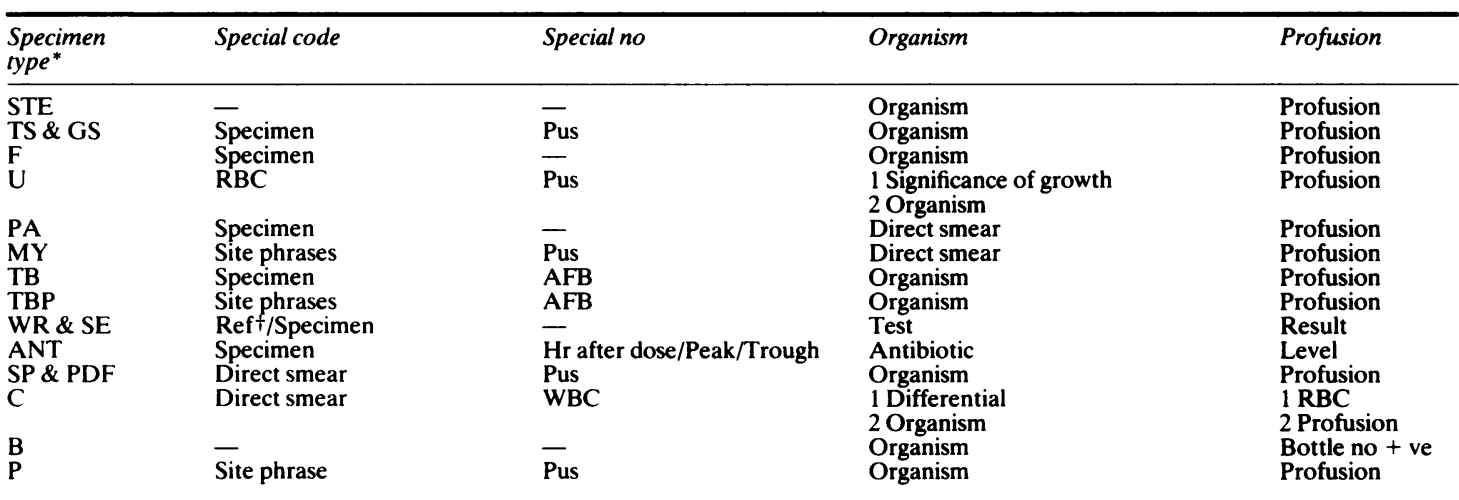

${ }^{*} \mathrm{STE}=$ sterility test TS = throat swab; GS = gynaecological swab; $\mathrm{F}=$ faeces $\mathrm{U}=$ urine; $\mathrm{PA}=$ parasitology; $\mathrm{MY}=\mathrm{mycology} ; \mathrm{ANT}=$ antibiotic assay; $\mathrm{SP}=$ sputum; $\mathrm{C}=$ cerebrospinal fluid; $\mathrm{TB}=$ sputum or urine for mycobacteria; $\mathrm{TBP}=$ pus, fluid, tissue etc for mycobacteria; WR = serological test for syphilis; $\mathrm{SE}=$ other serological test.

$\doteqdot R$ ef $=$ reference laboratory report.

code A0 to A8. These codes refer to nine rubber stamps in use in the laboratory. One such stamp used for staphylococci has the following layout: A1 PEN TET ERY CLI FUC CLX GEN NEO TOB. The technician then only has to fill in the spaces on the coding sheet with $\mathrm{S}, \mathrm{M}$, or $\mathrm{R}$. When the clerical staff enter this result they first type A1. This brings up the nine antibiotic headings as shown. They then enter the results in a form such as S S M R . . . all on one line, reducing the key entries from 54 to 21 . Each organism can have up to 20 sensitivity test results.

\section{STANDARD ENTRIES}

Approximately $45 \%$ of bacteriology results, mainly the negative specimens, have a standard format. Thus a normal urine would produce a record of the following type:

Special code

Special number

RBC 0

Organism

PUS 0

No bacterial growth

All these entries are made by the results program if the standard entry code $\mathrm{S} 1$ is entered in the special code position. There are 24 of these standard entries, so nearly half of the results can be entered by using one of a series S1 to S24 in answer to the special code question.

\section{COMPARISON WITH CHEMICAL PATHOLOGY}

Chemistry and haematology specimens have one result per test which is usually numerical and within a fixed range. As has been shown, bacteriology results may be short if the specimen is negative but allowance must be made for up to 10 tests in the organism position, and up to 20 sensitivity tests for an organism. It is obvious that the clinical file request record layout designed for chemical pathology will not cope with these needs. We have therefore designed our own layout and have added the facility of two lines of 56 characters each for comment (Figure).

\section{WRITING RESULTS DIRECTLY ONTO THE CLINICAL FILE}

Because of the size of bacteriology results and the need for work file records to be compatible in size with those of other disciplines, the work file was designed as a series of linked records, one record for $\mathbb{\perp}$ each organism in each specimen. One specimen could $\vec{\Rightarrow}$ thus result in up to 10 work file records. This meant $\stackrel{3}{3}$ that multiple file accesses were made when entering the results, when printing them, and again when removing them at authorisation. To avoid this unnecessary work, bacteriology results are written directly onto the clinical file. The work file record is simply marked to say that the result has been entered and a clinical file marker shows that the result is not authorised. Authorisation of a result involves deleting only one record from the work file and removing the marker from the clinical file.

As well as saving computer time, this method has the advantage that all results are immediately $N$ available for display within the laboratory, with a warning message if they are not yet authorised. They are not available on the ward VDUs until after authorisation.

FURTHER REPORT TO FOLLOW

Another area in which bacteriology differs from chemical pathology is that it is very rarely necessary to add to a chemical pathology result once it has been $\frac{\mathrm{O}}{\mathrm{D}}$ entered, while bacteriology results often require a $\stackrel{\bigcirc}{\circ}$ "further report to follow." The extreme case is for $\stackrel{\mathbb{Q}}{\square}$ 
Word

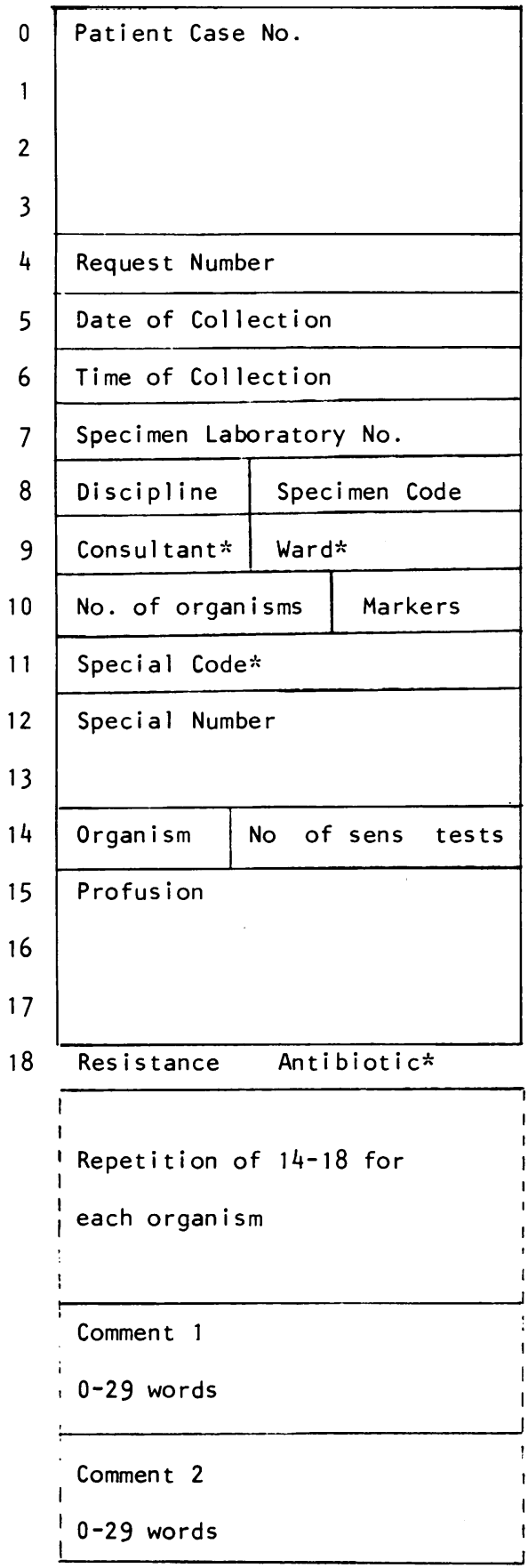

Clinical file request record. Asterisked items are translated into numbers from the index. Words $0-4$ are record key; words $0-8$ standard to Phoenix system; word 18 is repeated for each sensitivity test. tuberculosis specimens where eight weeks or more can elapse between specimen receipt and final report. If we know there is a further report to follow we enter the fact with the first result, and the work file record is flagged to this effect. Occasionally further results are produced for a specimen which was not marked in this way, so a facility was included to access the result on the clinical file and set up the work file record again.

\section{AUTHORISATION OF RESULTS}

Another difference in the running of the laboratories is in the concept of authorisation. Chemical Pathology list their results in sets of tests which are then passed to the laboratory staff responsible for those tests. When they are satisfied with the list all results in the set are authorised. In Bacteriology a member of the medical staff checks the results list against the handwritten results on the original request forms for each patient, which may contain more than one specimen type. Further there are often multiple single requests for various sites from one patient which from the point of view of clinical significance need to be checked together. We therefore rejected the system of authorising by specimen type (the equivalent of Chemical Pathology's set). Instead all results entered in a working day are printed in alphabetical order of patient surname. The request forms are also sorted alphabetically to facilitate checking and afterwards filed by patient name.

TIME TAKEN TO PROCESS SPECIMENS

A major difference between the disciplines is in the time taken to process specimens. Most Chemical Pathology specimens are completed within $24 \mathrm{~h}$ of the request being received. Bacteriological specimens may remain in the laboratory for days or even weeks awaiting results. With such delays it is possible to lose a specimen or a request form or to omit to enter the final result. We therefore print two lists. A daily printout of all specimens in laboratory number order shows from which patient any specimen comes. A delayed results list is also printed every week by looking at every record on the workfile. If a result has not been entered after a defined time interval for that specimen type, then that request is included on the list which the clerical staff checks.

\section{CUMULATIVE REPORTING}

The concept of cumulative reporting used by Chemical Pathology is not applicable to Bacteriology. For example a final tuberculosis result which would produce an unknown number of print lines may not be available for many weeks. Meanwhile further specimens and results for the same patient have taken us on to the next report page. We cannot just print the earlier page under the heading "file permanently in 
notes" because it is not yet complete. Therefore we initiated a system to give us twice daily reports listing all new results for each patient, and weekly review reports. The review contains all tuberculosis results still on the clinical file-retrospective up to one year-and all other results for the previous three months.

\section{ADDITIONAL PATIENT MARKERS}

Chemical Pathology have special flags on patients who are fee-paying or who are known to be HBsAg positive. We have added further flags for previous positive urine specimens, positive tuberculosis specimens and positive venereal serology results which are automatically set by the results program on entry. The urine marker is removed after six weeks but the positive VD serology marker causes the PID record to stay on the clinical file for five years which is useful in assessing the significance of current VD serology results whether positive or negative. We also have a marker for "medical interest," entered and removed by medical staff, for seriously ill patients whose requests and results are to be brought to the notice of the medical staff immediately.

\section{System performance}

The bacteriology system has operated since July 1980 . It was immediately obvious that the extra workload was too much for the computer to handle. The VDU response time was unacceptably long and as a result all disciplines were producing their reports far too late. Steps were taken to improve the situation. The memory of the computer was increased by Computer Technology Limited, another disc drive was added and a new operating system introduced. All these modifications made the working of the system faster. Night-time spooling, allowing programs to run unattended, was introduced for all reports and monthly statistics. Writing results directly to the clinical file also saved time. As a result the daytime capacity increased and we were able to start printing bacteriology reports at lunchtime as well as in the morning, thus bringing us back to the timetable we had achieved with the Elliott system, the majority of simple reports leaving the laboratory by $2 \mathrm{pm}$ on the day after receipt of the specimen.

\section{WORKLOAD}

The laboratory now has a workload of 64000 specimens per year. These requests and results are entered by two full-time and two part-time clerical staff.

All the coding of results on the request forms is done by the technicians. This work is then checked by the medical team and passed to the clerical staff for data entry. All technicians are trained in the use of the computer and know how to enter requests and results if necessary.

There are six VDUs in the Department. Two are $\frac{7}{0}$ used by the clerical staff for data entry and answering $\overline{0}$ telephone queries, one by the medical supervisory क team, and two are on the benches for the technical $\widehat{\nabla}$ staff to use for recalling previous results. The sixth is used by the part-time programmer who wrote the ${ }^{\infty}$ programs and maintains the bacteriology section of $\vec{O}$ the system.

The computer down-time is very small but in case ${ }^{\omega}$ of problems we have a minimal back-up system with which to answer enquiries. This consists of $(i)$ a day book in alphabetical order of surname so that we can $\%$ check that a particular patient's specimen has arrived; $\vec{\overrightarrow{ }}$ (ii) a listing of the day's results also in alphabetical $\vec{p}$ order; (iii) the request forms, with the encoded 0 results handwritten on them, kept filed for three months.

\section{Discussion}

Technically the adapted Phoenix system is far more $\vec{\oplus}$ sophisticated than the Elliott system. The main $\stackrel{\infty}{0}$ advantages include simplicity of data entry, the immediate detection and correction of most typing errors, easy editing of results when the bacteriology is incorrect and the immediate recall of results both in the laboratory and on the wards.

The main disadvantage is that when the computer $\stackrel{\square}{a}$ is down the clerical staff cannot continue data entry as they could with paper tape entry on the Elliott system. It is possible to enter patient identity details using paper tape for which Phoenix has a facility but this has not proved an attractive option and during응 down-time important results are telephoned to the $\overline{0}$ wards. Another disadvantage is that the limit on the number of peripheral devices attached to the $\delta$ computer is too small. It would be useful to have at $₹$ least two more VDUs, one for the Tuberculosis 을 laboratory and one dedicated for those answering the telephone enquiries. It would also be helpful to have a printer in the Bacteriology Department.

Possible future developments might include net- $\sigma$ working separate microsystems so that all the work $N$ does not have to pass through the same central $\mathbb{C}^{N}$ processing unit. Patient details and completed results would still be held on a common file.

Now that 18 months' results are on Phoenix, we are $\frac{\mathscr{C}}{\mathscr{D}}$ starting to analyse the data. There is a general $\stackrel{\infty}{?}$ purpose analysis program available to all laboratory 7 staff. They can specify a date range, ward, consultant, $\stackrel{\circ}{\circ}$

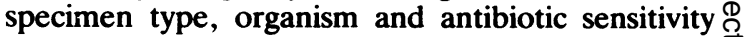
and all specimens satisfying these criteria are listed. $\mathbb{D}$ This can be used for checking occurrences of 
organisms in specific wards and for tracing the spread of an infection. It can also detect increasing resistance of a species to a particular antibiotic. Other analyses in progress include a summary of preoperative and postoperative results in surgical patients to detect operative infection and a study of the effectiveness of various combinations of antibiotics in the treatment of infection in neutropenic patients.

This is one of a small number of functioning clinical bacteriology reporting and analysis systems. It has been operating for over two years during which period all the software has been extensively tested. The number of integrated clinical pathology systems in Britain is even smaller; one such system has been recently introduced at University College Hospital.

We thank Professor IDP Wootton and his staff in the Department of Chemical Pathology, Royal
Postgraduate Medical School, and Austin Prall, previously of that department, now with Computer Technology Limited. We also thank Miss Avril Kirk for typing the manuscript.

\section{References}

' Mitchison DA, Darrell JH, Mitchison R. A computer-assisted bacteriology reporting and information system. J Clin Pathol 1978;31:673-80.

${ }^{2}$ Abson J, Prall A, Wootton IDP. Data processing in pathology laboratories: the Phoenix system. Anal Clin Biochem 1977;14:307-29.

Requests for reprints to: Dr JH Darrell, Department of Bacteriology, Royal Postgraduate Medical School, Hammersmith Hospital, Ducane Road, London W12 0HS, England. 